\title{
STABILITY OF MICROSTRUCTURE FOR TETRAGONAL TO MONOCLINIC MARTENSITIC TRANSFORMATIONS *
}

\author{
PAVEl BĚLÍK ${ }^{1}$ AND Mitchell Luskin ${ }^{1}$
}

\begin{abstract}
We give an analysis of the stability and uniqueness of the simply laminated microstructure for all three tetragonal to monoclinic martensitic transformations. The energy density for tetragonal to monoclinic transformations has four rotationally invariant wells since the transformation has four variants. One of these tetragonal to monoclinic martensitic transformations corresponds to the shearing of the rectangular side, one corresponds to the shearing of the square base, and one corresponds to the shearing of the plane orthogonal to a diagonal in the square base. We show that the simply laminated microstructure is stable except for a class of special material parameters. In each case that the microstructure is stable, we derive error estimates for the finite element approximation.
\end{abstract}

Mathematics Subject Classification. 49J45, 65N15, 65N30, 73C50, 73G05, 73K20, 73V05.

Received: March 29, 1999. Revised: November 25, 1999.

\section{INTRODUCTION}

We use the geometrically nonlinear theory of martensite $[2,3,13,28]$ to model tetragonal to monoclinic martensitic transformations. In this theory, the energy density is minimized on multiple energy wells $\mathrm{SO}(3) U_{1} \cup$ $\ldots \cup \mathrm{SO}(3) U_{N}$ where $U_{1}, \ldots, U_{N}$ for $N>1$ are symmetry-related transformation strains (variants) and $\mathrm{SO}(3)$ is the set of all $3 \times 3$ real orthogonal matrices with determinant equal to one. For tetragonal to monoclinic transformations, there are four symmetry-related transformation strains $(N=4)[34,35]$. There are three tetragonal to monoclinic martensitic transformations - one corresponds to shearing of a rectangular face, one corresponds to shearing of the square base, and one corresponds to shearing of the plane orthogonal to the diagonal of the square base. For certain boundary constraints or loading conditions, the elastic energy of a martensitic crystal is minimized only by the fine-scale mixing of deformation gradients from distinct energy wells. The simplest example of such a microstructure is the laminate in which two compatible deformation gradients oscillate in parallel layers of fine scale. Much recent work has been done to describe more complex microstructures by using the concept of the Young measure [2,3,21,36,37].

The stability theory that we use was first used to study the orthorhombic to monoclinic transformation $(N=2)[27]$. It was then extended to obtain results for the cubic to tetragonal transformation $(N=3)[23]$. Most recently, the stability theory has been used to analyze a cubic to orthorhombic transformation $(N=6)[6]$.

Keywords and phrases. Martensitic transformation, microstructure, nonconvex variational problem, simple laminate, tetragonal, monoclinic, volume fraction, Young measure, finite element, error estimate

* This work was supported in part by NSF DMS 95-05077, by AFOSR F49620-98-1-0433, by ARO DAAG55-98-1-0335, by the Institute for Mathematics and Its Applications, and by the Minnesota Supercomputer Institute.

${ }^{1}$ School of Mathematics, University of Minnesota, 206 Church Street SE, Minneapolis, MN 55455, USA; e-mail: belik@math.umn.edu \& luskin@math.umn.edu 
In general, the analysis of stability is more difficult for transformations with $N=4$ (such as the tetragonal to monoclinic transformations studied in this paper) and $N=6$ since the additional wells give the crystal more freedom to deform without the cost of additional energy. In fact, we show here that there are special lattice constants for which the simply laminated microstructure for the tetragonal to monoclinic transformation is not stable.

The stability theory can also be used to analyze laminates with varying volume fraction [24] and conforming and nonconforming finite element approximations [25,27]. We also note that the stability theory was used to analyze the microstructure in ferromagnetic crystals [29]. Related results on the numerical analysis of nonconvex variational problems can be found, for example, in [7-12, 14-16, 18, 19, 22, 26, 30-33].

In this paper, we give an analysis of the stability of a laminated microstructure with infinitesimal length scale that oscillates between two compatible variants. We show that for any other deformation satisfying the same boundary conditions as the laminate, we can bound the pertubation of the volume fractions of the variants by the pertubation of the bulk energy. This implies that the volume fractions of the variants for a deformation are close to the volume fractions of the laminate if the bulk energy of the deformation is close to the bulk energy of the laminate. This concept of stability can be applied directly to obtain results on the convergence of finite element approximations and guarantees that any finite element solution with sufficiently small bulk energy gives reliable approximations of the stable quantities such as volume fraction.

In Section 2, we describe the geometrically nonlinear theory of martensite. We refer the reader to [2,3] and to the introductory article [28] for a more detailed discussion of the geometrically nonlinear theory of martensite. We review the results given in $[34,35]$ on the transformation strains and possible interfaces for tetragonal to monoclinic transformations corresponding to the shearing of the square and rectangular faces, and we then give the transformation strain and possible interfaces corresponding to the shearing of the plane orthogonal to a diagonal in the square base.

In Section 3, we give the main results of this paper which give bounds on the volume fraction of the crystal in which the deformation gradient is in energy wells that are not used in the laminate. These estimates are used in Section 4 to establish a series of error bounds in terms of the elastic energy of deformations for the $\mathrm{L}^{2}$ approximation of the directional derivative of the limiting macroscopic deformation in any direction tangential to the parallel layers of the laminate, for the $\mathrm{L}^{2}$ approximation of the limiting macroscopic deformation, for the approximation of volume fractions of the participating martensitic variants, and for the approximation of nonlinear integrals of deformation gradients. Finally, in Section 5 we give an application of the stability theory to the finite element approximation of the simply laminated microstructure.

\section{ThE GEOMETRICALLY NONLINEAR MODEL}

We use the austenitic tetragonal phase of the crystal at the transformation temperature as the reference configuration $\Omega \subset \mathbb{R}^{3}$, and we assume that $\Omega$ is a bounded domain with a Lipschitz continuous boundary $\partial \Omega$. We denote deformations by functions $y: \Omega \rightarrow \mathbb{R}^{3}$ and corresponding deformation gradients by $\nabla y: \Omega \rightarrow \mathbb{R}^{3 \times 3}$ where $\mathbb{R}^{3 \times 3}$ denotes the set of all $3 \times 3$ real matrices.

We shall minimize the total energy

$$
\mathcal{E}(y)=\int_{\Omega} \phi(\nabla y(x)) \mathrm{d} x
$$

over an admissible class $\mathcal{A}$ of deformations, where $\phi: \mathbb{R}^{3 \times 3} \rightarrow \mathbb{R}$ is the free energy density per unit volume of the reference configuration of the crystal at a fixed temperature below the transformation temperature.

We shall assume that the free energy density is frame-indifferent, that is,

$$
\phi(R F)=\phi(F) \quad \text { for all } F \in \mathbb{R}^{3 \times 3} \text { and } R \in \mathrm{SO}(3) \text {. }
$$


We also assume that it inherits the symmetry of the austenitic phase of the crystal, so that

$$
\phi\left(R_{i}^{T} F R_{i}\right)=\phi(F) \quad \text { for all } F \in \mathbb{R}^{3 \times 3} \text { and } R_{i} \in \mathcal{G},
$$

where $\mathcal{G}=\left\{R_{1}, \ldots, R_{8}\right\} \subset \mathrm{SO}(3)$ is the symmetry group of the tetragonal phase given by

$$
\begin{gathered}
R_{1}=R\left(\pi, e_{1}\right), \quad R_{2}=R\left(\pi, e_{2}\right), \quad R_{3}=R\left(\pi, e_{3}\right), \\
R_{4}=R\left(\pi, e_{1}+e_{2}\right), \quad R_{5}=R\left(\pi, e_{1}-e_{2}\right), \\
R_{6}=R\left(\pi / 2, e_{3}\right), \quad R_{7}=R\left(3 \pi / 2, e_{3}\right), \quad R_{8}=I .
\end{gathered}
$$

In the above, $\left\{e_{i}\right\}$ is a right-handed, orthonormal basis for $\mathbb{R}^{3}$ given by normalized lattice vectors for the tetragonal phase, and $R(\alpha, v)$ denotes the rotation of $\alpha$ radians about $v \in \mathbb{R}^{3}, v \neq 0$.

We assume that the free energy density is minimized at a transformation (Bain) strain $U_{1}$ for the tetragonal to monoclinic transformation. We shall see that there then exist three other distinct symmetry-related transformation strains (variants) $U_{2}, U_{3}, U_{4}$ such that

$$
\left\{R_{i}^{T} U_{1} R_{i}: R_{i} \in \mathcal{G}\right\}=\left\{U_{1}, \ldots, U_{4}\right\} .
$$

It also follows by the frame-indifference (2.1) and the symmetry (2.2) of the energy density that the energy density is minimized on the union $\mathcal{U}$ of the four energy wells

$$
\mathcal{U}_{i}=\mathrm{SO}(3) U_{i}=\left\{R U_{i}: R \in \mathrm{SO}(3)\right\} \quad \text { for } i=1, \ldots, 4 .
$$

By adding a constant, we may assume that the minimum value of $\phi$ is 0 . Finally, we shall assume that $\phi$ is continuous and satisfies the growth condition

$$
\phi(F) \geq \kappa\|F-\pi(F)\|^{2} \quad \text { for all } F \in \mathbb{R}^{3 \times 3},
$$

where $\kappa>0$ is a constant and $\pi: \mathbb{R}^{3 \times 3} \rightarrow \mathcal{U}$ is a projection defined by

$$
\|F-\pi(F)\|=\min _{G \in \mathcal{U}}\|F-G\| \quad \text { for all } F \in \mathbb{R}^{3 \times 3} .
$$

This projection exists for any $F \in \mathbb{R}^{3 \times 3}$, since the set $\mathcal{U}$ is compact.

We now derive the transformation strains for the three tetragonal to monoclinic transformations. The reader should note that this derivation itself is not used in the stability analysis given below; only the resulting transformation strains described in (2.6), (2.7), and (2.8) will be used.

Each of the two-fold rotations $R_{l}$ for $l=1, \ldots, 5$ in the tetragonal symmetry group determines a family of transformation strains that corresponds to shearing in the plane orthogonal to the axis of the rotation. For each two-fold rotation $R_{l}$ for $l=1, \ldots, 5$, the transformation strains $U_{1}^{(l)}$ in the corresponding family satisfy $[3,34,35]$

$$
\left\{R_{i} \in \mathcal{G}: R_{i}^{T} U_{1}^{(l)} R_{i}=U_{1}^{(l)}\right\}=\left\{I, R_{l}\right\} .
$$

The corresponding symmetry-related variants $U_{2}^{(l)}, U_{3}^{(l)}, U_{4}^{(l)}$ are then given by

$$
\left\{R_{i}^{T} U_{1}^{(l)} R_{i}: R_{i} \in \mathcal{G}\right\}=\left\{U_{1}^{(l)}, \ldots, U_{4}^{(l)}\right\} .
$$

The two-fold rotations $R_{1}$ and $R_{2}$ correspond to shearing of the rectangular faces and can be analyzed identically by symmetry. The two-fold rotation $R_{3}$ corresponds to shearing of the square base and must be treated as a separate case. The two-fold rotations $R_{4}$ and $R_{5}$ correspond to shearing the plane orthogonal to a diagonal 
of the square base and can also be analyzed identically by symmetry. Therefore, we need analyze only the following three cases corresponding to $R_{2}, R_{3}$, and $R_{4}$.

In Case 1, corresponding to $R_{2}$ and shearing in the plane orthogonal to $e_{2}$, the transformation strain $U_{1}^{(2)}$ and the symmetry-related transformation strains $U_{2}^{(2)}, U_{3}^{(2)}, U_{4}^{(2)}$ are given by

$$
\begin{array}{rlrl}
U_{1}^{(2)} & =\left(\begin{array}{ccc}
\theta_{1} & 0 & \theta_{4} \\
0 & \theta_{2} & 0 \\
\theta_{4} & 0 & \theta_{3}
\end{array}\right), & U_{2}^{(2)}=\left(\begin{array}{ccc}
\theta_{1} & 0 & -\theta_{4} \\
0 & \theta_{2} & 0 \\
-\theta_{4} & 0 & \theta_{3}
\end{array}\right), \\
U_{3}^{(2)}=\left(\begin{array}{ccc}
\theta_{2} & 0 & 0 \\
0 & \theta_{1} & \theta_{4} \\
0 & \theta_{4} & \theta_{3}
\end{array}\right), & U_{4}^{(2)}=\left(\begin{array}{ccc}
\theta_{2} & 0 & 0 \\
0 & \theta_{1} & -\theta_{4} \\
0 & -\theta_{4} & \theta_{3}
\end{array}\right),
\end{array}
$$

where $\theta_{i}>0$ for $i=1,2,3, \theta_{4} \neq 0$, and $\theta_{1} \theta_{3}-\theta_{4}^{2}>0$. We shall assume without loss of generality that $\theta_{4}>0$.

For Case 2, corresponding to $R_{3}$ and shearing in the plane orthogonal to $e_{3}$, the transformation strain $U_{1}^{(3)}$ and the symmetry-related transformation strains $U_{2}^{(3)}, U_{3}^{(3)}, U_{4}^{(3)}$ are given by

$$
\begin{array}{rlrl}
U_{1}^{(3)} & =\left(\begin{array}{ccc}
\delta_{1} & \delta_{4} & 0 \\
\delta_{4} & \delta_{2} & 0 \\
0 & 0 & \delta_{3}
\end{array}\right), & U_{2}^{(3)} & =\left(\begin{array}{ccc}
\delta_{1} & -\delta_{4} & 0 \\
-\delta_{4} & \delta_{2} & 0 \\
0 & 0 & \delta_{3}
\end{array}\right), \\
U_{3}^{(3)} & =\left(\begin{array}{ccc}
\delta_{2} & \delta_{4} & 0 \\
\delta_{4} & \delta_{1} & 0 \\
0 & 0 & \delta_{3}
\end{array}\right), & U_{4}^{(3)}=\left(\begin{array}{ccc}
\delta_{2} & -\delta_{4} & 0 \\
-\delta_{4} & \delta_{1} & 0 \\
0 & 0 & \delta_{3}
\end{array}\right),
\end{array}
$$

where $\delta_{i}>0$ for $i=1,2,3, \delta_{4} \neq 0$ and $\delta_{1} \delta_{2}-\delta_{4}^{2}>0$. We shall assume without loss of generality that $\delta_{1}>\delta_{2}$ and $\delta_{4}>0$.

In Case 3, corresponding to $R_{4}$ and shearing in the plane orthogonal to $e_{1}+e_{2}$, the transformation strain $U_{1}^{(4)}$ and the symmetry-related transformation strains $U_{2}^{(4)}, U_{3}^{(4)}, U_{4}^{(4)}$ are given by

$$
\begin{array}{rlrl}
U_{1}^{(4)} & =\left(\begin{array}{ccc}
\eta_{1} & \eta_{3} & \eta_{4} \\
\eta_{3} & \eta_{1} & -\eta_{4} \\
\eta_{4} & -\eta_{4} & \eta_{2}
\end{array}\right), & U_{2}^{(4)}=\left(\begin{array}{ccc}
\eta_{1} & -\eta_{3} & \eta_{4} \\
-\eta_{3} & \eta_{1} & \eta_{4} \\
\eta_{4} & \eta_{4} & \eta_{2}
\end{array}\right), \\
U_{3}^{(4)}=\left(\begin{array}{ccc}
\eta_{1} & \eta_{3} & -\eta_{4} \\
\eta_{3} & \eta_{1} & \eta_{4} \\
-\eta_{4} & \eta_{4} & \eta_{2}
\end{array}\right), & U_{4}^{(4)}=\left(\begin{array}{ccc}
\eta_{1} & -\eta_{3} & -\eta_{4} \\
-\eta_{3} & \eta_{1} & -\eta_{4} \\
-\eta_{4} & -\eta_{4} & \eta_{2}
\end{array}\right),
\end{array}
$$

where $\eta_{1}>0, \eta_{2}>0, \eta_{4} \neq 0, \eta_{1}+\eta_{3}>0$, and $\eta_{2}\left(\eta_{1}-\eta_{3}\right)-2 \eta_{4}^{2}>0$. We shall assume without loss of generality that $\eta_{4}>0$ (Note that it follows from the preceding inequality that $\eta_{1}+\eta_{2}-\eta_{3}>0$ and $\eta_{1} \eta_{2}-\eta_{4}^{2}>0$; we will use these inequalities in the proof of Theorem 3.1).

In what follows, we will omit the superscript in the notation for the transformation strain $U_{i}^{(l)}$ since the case being considered will always be explicitly given.

There exists a continuous deformation $y(x) \in \mathrm{C}^{0}\left(\mathbb{R}^{3} ; \mathbb{R}^{3}\right)$ such that $[2,28]$

$$
\nabla y(x)= \begin{cases}Q U_{i} & \text { for all } x \text { such that } x \cdot n<s, \\ U_{j} & \text { for all } x \text { such that } x \cdot n>s,\end{cases}
$$

where $Q \in \mathrm{SO}(3), i, j \in\{1, \ldots, 4\}, n \in \mathbb{R}^{3}, n \neq 0$, and $s \in \mathbb{R}$, if and only if there exists $a \in \mathbb{R}^{3}$ such that

$$
Q U_{i}=U_{j}+a \otimes n .
$$


Thus, if (2.9) holds for $a \neq 0$, then $x \cdot n=s$ is an interface plane; and we say that the two wells $\mathcal{U}_{i}=\operatorname{SO}(3) U_{i}$ and $\mathcal{U}_{j}=\mathrm{SO}(3) U_{j}$ are rank-one connected.

The following lemma (which is a special case of Proposition 2.2 in [4]) will be used to construct rank-one connections for the tetragonal to monoclinic transformation that result from the two-fold rotations in the symmetry group. This lemma can be verified by direct substitution into (2.9).

Lemma 2.1. Assume that $U_{i}, U_{j} \in \mathbb{R}^{3 \times 3}$ are positive definite and symmetric, that there exists a unit vector $m \in \mathbb{R}^{3}$, and a rotation $R(\pi, m) \in \mathcal{G}$ such that

$$
U_{i}=R(\pi, m)^{T} U_{j} R(\pi, m)
$$

Then there exist exactly two solutions to (2.9), up to the scaling of a and $n$ by any nonzero constant $\rho \in \mathbb{R}$, given by

$$
a=\frac{2}{\rho}\left(\frac{U_{j}^{-1} m}{\left|U_{j}^{-1} m\right|^{2}}-U_{j} m\right), \quad n=\rho m, \quad Q=R\left(\pi, U_{j}^{-1} n\right) R(\pi, m),
$$

and

$$
a=\rho U_{j} m, \quad n=\frac{2}{\rho}\left(m-\frac{U_{j}^{2} m}{\left|U_{j} m\right|^{2}}\right), \quad Q=R(\pi, a) R(\pi, m) .
$$

The first solution given in Lemma 2.1 determines a type $I$ twin and the other solution determines a type $I I$ twin $[2,38]$. For either type of twin, we call the planes $x \cdot n=s$ twin planes. If a twin is both a type I twin and a type II twin, then it is said to be a compound twin. The following easily proven identities give type I twins and type II twins by the above lemma.

Lemma 2.2. The two-fold rotations defined in $(2.3)$ act on $U_{1}, \ldots, U_{4}$ in the following manner:

Case 1:

$$
\begin{array}{ll}
R_{1}^{T} U_{1} R_{1}=R_{3}^{T} U_{1} R_{3}=U_{2}, & R_{5}^{T} U_{2} R_{5}=U_{4}, \\
R_{2}^{T} U_{1} R_{2}=U_{1}, & R_{4}^{T} U_{2} R_{4}=U_{3}, \\
R_{4}^{T} U_{1} R_{4}=U_{4}, & R_{1}^{T} U_{3} R_{1}=U_{3}, \\
R_{5}^{T} U_{1} R_{5}=U_{3}, & R_{2}^{T} U_{3} R_{2}=R_{3}^{T} U_{3} R_{3}=U_{4}, \\
R_{2}^{T} U_{2} R_{2}=U_{2}, & R_{1}^{T} U_{4} R_{1}=U_{4} .
\end{array}
$$

Case 2:

$$
\begin{array}{ll}
R_{1}^{T} U_{1} R_{1}=R_{2}^{T} U_{1} R_{2}=U_{2}, & R_{4}^{T} U_{2} R_{4}=R_{5}^{T} U_{2} R_{5}=U_{4}, \\
R_{3}^{T} U_{1} R_{3}=U_{1}, & R_{1}^{T} U_{3} R_{1}=R_{2}^{T} U_{3} R_{2}=U_{4}, \\
R_{4}^{T} U_{1} R_{4}=R_{5}^{T} U_{1} R_{5}=U_{3}, & R_{3}^{T} U_{3} R_{3}=U_{3}, \\
R_{3}^{T} U_{2} R_{3}=U_{2}, & R_{3}^{T} U_{4} R_{3}=U_{4} .
\end{array}
$$

Case 3:

$$
\begin{array}{ll}
R_{1}^{T} U_{1} R_{1}=U_{4}, & R_{3}^{T} U_{2} R_{3}=R_{4}^{T} U_{2} R_{4}=U_{4}, \\
R_{2}^{T} U_{1} R_{2}=U_{2}, & R_{5}^{T} U_{2} R_{5}=U_{2} \\
R_{3}^{T} U_{1} R_{3}=R_{5}^{T} U_{1} R_{5}=U_{3}, & R_{2}^{T} U_{3} R_{2}=U_{4} \\
R_{4}^{T} U_{1} R_{4}=U_{1}, & R_{4}^{T} U_{3} R_{4}=U_{3} \\
R_{1}^{T} U_{2} R_{1}=U_{3}, & R_{5}^{T} U_{4} R_{5}=U_{4}
\end{array}
$$

We next use the above lemma to give the following result classifying all possible rank-one connections for the tetragonal to monoclinic transformations. We note that for Case 2 there exist rank-one connections that are neither type I nor type II. The interfaces separating such rank-one connections are sometimes called domain interfaces rather than twin interfaces [20], and we shall maintain this distinction below. 
Lemma 2.3. For Case 1, Case 2, and Case 3, we have:

1. For each $i \in\{1, \ldots, 4\}$, the energy well $\mathcal{U}_{i}$ is not rank-one connected to itself.

2. For any $i, j \in\{1, \ldots, 4\}$, with $i \neq j$, there are exactly two solutions to the twinning equation (2.9). The characterization of the solutions to (2.9) is given in Table 1 where

$$
\varepsilon_{1}=\frac{\theta_{2}^{2}-\theta_{1}^{2}-\theta_{4}^{2}}{\left[2\left(\theta_{2}^{2}-\theta_{1}^{2}-\theta_{4}^{2}\right)^{2}+4 \theta_{4}^{2}\left(\theta_{1}+\theta_{3}\right)^{2}\right]^{1 / 2}}, \quad \zeta=\arctan \frac{\delta_{1}-\delta_{2}}{2 \delta_{4}}
$$

and

$$
\varepsilon_{2}=\frac{2 \eta_{1} \eta_{3}-\eta_{4}^{2}}{\left[\left(2 \eta_{1} \eta_{3}-\eta_{4}^{2}\right)^{2}+\eta_{4}^{2}\left(\eta_{1}+\eta_{2}-\eta_{3}\right)^{2}\right]^{1 / 2}}
$$

Alternatively,

$$
\cos \frac{\zeta}{2}=\left[\frac{1}{2}+\frac{\delta_{4}}{\sqrt{4 \delta_{4}^{2}+\left(\delta_{1}-\delta_{2}\right)^{2}}}\right]^{1 / 2}, \quad \sin \frac{\zeta}{2}=\left[\frac{1}{2}-\frac{\delta_{4}}{\sqrt{4 \delta_{4}^{2}+\left(\delta_{1}-\delta_{2}\right)^{2}}}\right]^{1 / 2} .
$$

Proof. There do not exist $R_{0}, R_{1} \in \mathrm{SO}(3)$ with $R_{0} \neq R_{1}$ and $a, n \in \mathbb{R}^{3}, a, n \neq 0$ such that $[2,28]$

$$
R_{1}=R_{0}+a \otimes n
$$

Hence, for each $i \in\{1, \ldots, 4\}$, the energy well $\mathcal{U}_{i}$ is not rank-one connected to itself.

By Lemma 2.2, all of the interfaces in Case 1 and Case 3 and all but the $(i, j)=(1,4)$ or $(i, j)=(2,3)$ interfaces of Case 2 satisfy (2.10). Hence, Lemma 2.1 can be applied to obtain the solutions to (2.9).

To compute the interface normals for $(i, j)=(1,4)$ or $(i, j)=(2,3)$ in Case 2, we recall from Lemma 5 in [28] that $n=\left(\mu_{1}, \mu_{2}, 0\right), n \neq 0$, is an interface normal if and only if

$$
\left|U_{i} v\right|=\left|U_{j} v\right|
$$

for $v=\left(-\mu_{2}, \mu_{1}, 0\right)$.

In the following, we will be interested in a simple laminate. We fix $i, j \in\{1, \ldots, 4\}$ with $i \neq j$, and $Q, a$, and $n$ with $a, n \neq 0$ that satisfy the interface equation

$$
Q U_{i}=U_{j}+a \otimes n
$$

For any fixed $\lambda \neq 0,1$, we denote

$$
F_{\lambda}=\lambda Q U_{i}+(1-\lambda) U_{j}=U_{j}+\lambda a \otimes n
$$

Then we have the following lemma.

Lemma 2.4. For any $\lambda \in(0,1)$, we have that $F_{\lambda} \notin \mathcal{U}$.

Proof. It is proved in Lemma 2.3 of $[6]$ that $F_{\lambda} \notin \mathcal{U}$ if $(i, j)$ is a type I or type II twin. This proves the theorem in Case 1 and Case 3.

We next consider Case 2. If $F_{\lambda} \in \mathcal{U}$, then

$$
\lambda Q U_{i}+(1-\lambda) U_{j}=R U_{k}
$$


TABLE 1. Characterization of the solutions to (2.9).

\begin{tabular}{|c|c|c|c|}
\hline CASE & $\overline{(i, j)}$ & T"TYPE OF TWIN & INTERFACE NORMALS \\
\hline \multirow{12}{*}{ CASE 1} & $\begin{array}{l}(1,2) \\
\end{array}$ & compound & $\overline{n_{1}=e_{1}}$ \\
\hline & & compound & $n_{2}=e_{3}$ \\
\hline & $(3,4)$ & compound & $n_{1}=e_{2}$ \\
\hline & & compound & $n_{2}=e_{3}$ \\
\hline & $(1,3)$ & I & $e_{1}-e_{2}$ \\
\hline & & II & $\left(-\varepsilon_{1},-\varepsilon_{1}, \sqrt{1-2 \varepsilon_{1}^{2}}\right)$ \\
\hline & $(1,4)$ & $\mathrm{I}$ & $e_{1}+e_{2}$ \\
\hline & & II & $\left(-\varepsilon_{1}, \varepsilon_{1}, \sqrt{1-2 \varepsilon_{1}^{2}}\right)$ \\
\hline & $(2,3)$ & I & $e_{1}+e_{2}$ \\
\hline & & II & $\left(\varepsilon_{1},-\varepsilon_{1}, \sqrt{1-2 \varepsilon_{1}^{2}}\right)$ \\
\hline & $(2,4)$ & I & $e_{1}-e_{2}$ \\
\hline & & II & $\left(\varepsilon_{1}, \varepsilon_{1}, \sqrt{1-2 \varepsilon_{1}^{2}}\right)$ \\
\hline \multirow{12}{*}{ CASE 2} & $(1,2)$ & compound & $n_{1}=e_{2}$ \\
\hline & & compound & $e_{1}$ \\
\hline & $(1,3)$ & compound & $n_{1}=e_{1}-e_{2}$ \\
\hline & & compound & $e_{1}+e_{2}$ \\
\hline & $(2,4)$ & compound & $n_{1}=e_{1}+e_{2}$ \\
\hline & & compound & $e_{1}-e_{2}$ \\
\hline & $(3,4)$ & compound & $n_{1}=e_{1}$ \\
\hline & & compound & $e_{2}$ \\
\hline & $(1,4)$ & domain & $\left(\cos \frac{\zeta}{2},-\sin \frac{\zeta}{2}, 0\right)$ \\
\hline & & domain & $\left(\sin \frac{\zeta}{2}, \cos \frac{\zeta}{2}, 0\right)$ \\
\hline & $(2,3)$ & domain & $\left(\cos \frac{\xi}{2}, \sin \frac{\xi}{2}, 0\right)$ \\
\hline & & domain & $\left(\sin \frac{\zeta}{2},-\cos \frac{\zeta}{2}, 0\right)$ \\
\hline \multirow{12}{*}{ CASE 3} & $(1,2)$ & $\mathrm{I}$ & $e_{2}$ \\
\hline & & II & $\left(\varepsilon_{2}, 0,-\sqrt{1-\varepsilon_{2}^{2}}\right)$ \\
\hline & $(1,3)$ & compound & $n_{1}=e_{3}$ \\
\hline & & compound & $n_{2}=e_{1}-e_{2}$ \\
\hline & $(1,4)$ & I & $e_{1}$ \\
\hline & & II & $\left(0, \varepsilon_{2}, \sqrt{1-\varepsilon_{2}^{2}}\right)$ \\
\hline & $(2,3)$ & I & $e_{1}$ \\
\hline & & II & $\left(0,-\varepsilon_{2}, \sqrt{1-\varepsilon_{2}^{2}}\right)$ \\
\hline & $(2,4)$ & compound & $n_{1}=e_{3}$ \\
\hline & & compound & $n_{2}=e_{1}+e_{2}$ \\
\hline & $(3,4)$ & I & $e_{2}$ \\
\hline & & II & $\left(\varepsilon_{2}, 0, \sqrt{1-\varepsilon_{2}^{2}}\right)$ \\
\hline
\end{tabular}

for some $i \neq j$ and $k \in\{1, \ldots, 4\}$. Then by the interface equation (2.11) we have

$$
\begin{gathered}
Q U_{i}+(1-\lambda) a \otimes n=R U_{k}, \\
U_{j}+\lambda a \otimes n=R U_{k} .
\end{gathered}
$$

If $k=i$ or $k=j$, then (2.13) implies that the energy well $\mathcal{U}_{i}$ or $\mathcal{U}_{j}$ is rank-one connected to itself. This contradicts Lemma 2.3. If $k \neq i$ and $k \neq j$, then (2.13) implies that an interface normal for $(i, j)$ is the same 
as an interface normal for $(i, k)$ and $(j, k)$. This contradicts the table of interface normals for Case 2 given in Lemma 2.3.

We shall assume that the energy density $\phi(F)$ satisfies the growth condition

$$
\phi(F) \geq C_{1}\|F\|^{p}-C_{0} \quad \text { for all } F \in \mathbb{R}^{3 \times 3},
$$

where $C_{0}$ and $C_{1}$ are positive constants independent of $F \in \mathbb{R}^{3 \times 3}$ and where we assume $p>3$ to ensure that deformations with finite energy are uniformly continuous [1]. We can then denote the set of deformations of finite energy by

$$
W^{\phi}=\left\{y \in \mathrm{C}^{0}\left(\bar{\Omega} ; \mathbb{R}^{3}\right): \int_{\Omega} \phi(\nabla y(x)) \mathrm{d} x<\infty\right\},
$$

and we can define the set $\mathcal{A}$ of admissible deformations as

$$
\mathcal{A}=\left\{y \in W^{\phi}: y(x)=y_{0}(x) \text { for all } x \in \partial \Omega\right\}
$$

where

$$
y_{0}(x)=F_{\lambda} x \quad \text { for all } x \in \Omega
$$

We can prove the following lemma by constructing laminates with length scale converging to zero whose deformation gradients oscillate with volume fraction $\lambda$ at $Q U_{i}$ and $1-\lambda$ at $U_{j}[12,28]$.

Lemma 2.5. Let $\mathcal{A}$ be defined as in (2.14). Then the total energy $\mathcal{E}(y)$ satisfies

$$
\inf _{y \in \mathcal{A}} \mathcal{E}(y)=0
$$

\section{Reduction to the APproximate miXture of two STRAins}

Recall the definitions (2.5) and (2.14) of $\pi$ and $\mathcal{A}$, respectively. For each $k \in\{1, \ldots, 4\}$ and each $y \in \mathcal{A}$, we define

$$
\Omega_{k}(y)=\left\{x \in \Omega: \pi(\nabla y(x)) \in \mathcal{U}_{k}\right\}
$$

and the volume fraction with respect to the $k$-th energy well $\mathcal{U}_{k}$ to be

$$
\tau_{k}(y)=\frac{\operatorname{meas} \Omega_{k}(y)}{\operatorname{meas} \Omega}
$$

Since every $x \in \Omega$ is in $\Omega_{k}(y)$ for some $k \in\{1, \ldots, 4\}$, we have that

$$
\sum_{k=1}^{4} \tau_{k}(y)=1 \quad \text { for all } y \in \mathcal{A} \text {. }
$$

By the rank-one connection (2.11) and the definition of $F_{\lambda}$

$$
F_{\lambda}=\lambda Q U_{i}+(1-\lambda) U_{j}=U_{j}+\lambda a \otimes n
$$

we have that

$$
\left|F_{\lambda} w\right|=\left|U_{i} w\right|=\left|U_{j} w\right| \quad \text { for all } w \in \mathbb{R}^{3}, w \cdot n=0 \text {. }
$$


Since $\operatorname{det}\left(Q U_{i}\right)=\operatorname{det} U_{j}>0$, we have that $U_{j}^{-1} a \cdot n=0$. Hence, we have that

$$
\operatorname{Cof} F_{\lambda}=\left(\operatorname{Cof} U_{j}\right)\left(I-\lambda n \otimes U_{j}^{-1} a\right)
$$

where the cofactor of a nonsingular $A \in \mathbb{R}^{3 \times 3}$ is defined by $\operatorname{Cof} A=(\operatorname{det} A) A^{-T}$. We then obtain from (3.4) that

$$
\left|\left(\operatorname{Cof} F_{\lambda}\right) w\right|=\left|\left(\operatorname{Cof} U_{i}\right) w\right|=\left|\left(\operatorname{Cof} U_{j}\right) w\right| \quad \text { for all } w \in \mathbb{R}^{3}, w \cdot U_{j}^{-1} a=0 .
$$

We next recall that since the subdeterminant of the gradient is a null-Lagrangian [17], we have for $y \in \mathcal{A}$ that

$$
\begin{aligned}
\int_{\Omega} \nabla y(x) \mathrm{d} x & =\int_{\Omega} F_{\lambda} \mathrm{d} x \\
\int_{\Omega} \operatorname{Cof} \nabla y(x) \mathrm{d} x & =\int_{\Omega} \operatorname{Cof} F_{\lambda} \mathrm{d} x .
\end{aligned}
$$

Finally, we note that it follows from (2.4) that

$$
\int_{\Omega}\|\nabla y(x)-\pi(\nabla y(x))\|^{2} \mathrm{~d} x \leq \kappa^{-1} \mathcal{E}(y) \quad \text { for all } y \in \mathcal{A} .
$$

The following result is proved in more detail in [6] for the cubic to orthorhombic transformation. In the estimates below, $C$ will denote a generic positive constant that is independent of $y \in \mathcal{A}$ and is allowed to change from equation to equation.

Lemma 3.1. Given $i, j \in\{1, \ldots, 4\}, Q \in \mathrm{SO}(3)$, and $a, n \in \mathbb{R}, a, n \neq 0$ satisfying the twinning equation (2.11), there exists a constant $C>0$ such that for any $y \in \mathcal{A}$

$$
\begin{aligned}
\rho_{1}(y ; w) & \equiv \sum_{k \neq i, j} \tau_{k}(y)\left(\left|F_{\lambda} w\right|^{2}-\left|U_{k} w\right|^{2}\right) \\
& \leq C \mathcal{E}(y)^{1 / 2} \quad \text { for all } w \in \mathbb{R}^{3},|w|=1, w \cdot n=0, \\
\rho_{2}(y ; w) & \equiv \sum_{k \neq i, j} \tau_{k}(y)\left[\left|\operatorname{Cof}\left(F_{\lambda}\right) w\right|^{2}-\left|\left(\operatorname{Cof} U_{k}\right) w\right|^{2}\right] \\
& \leq C\left[\mathcal{E}(y)^{1 / 2}+\mathcal{E}(y)\right] \quad \text { for all } w \in \mathbb{R}^{3},|w|=1, w \cdot U_{j}^{-1} a=0 .
\end{aligned}
$$

Proof. We have by (3.1) and (3.6) that for any $w \in \mathbb{R}^{3}$ with $|w|=1$

$$
\begin{aligned}
\rho_{1}(y ; w)= & \sum_{k=1}^{4} \tau_{k}(y)\left(\left|F_{\lambda} w\right|^{2}-\left|U_{k} w\right|^{2}\right) \\
= & \frac{1}{\operatorname{meas} \Omega} \int_{\Omega}\left[\left|F_{\lambda} w\right|^{2}-|\pi(\nabla y(x)) w|^{2}\right] \mathrm{d} x \\
= & -\frac{1}{\operatorname{meas} \Omega} \int_{\Omega}\left|\left[F_{\lambda}-\pi(\nabla y(x))\right] w\right|^{2} \mathrm{~d} x \\
& +\frac{2}{\operatorname{meas} \Omega} \int_{\Omega}[\nabla y(x)-\pi(\nabla y(x))] w \cdot F_{\lambda} w \mathrm{~d} x \\
\leq & \frac{2}{\operatorname{meas} \Omega} \int_{\Omega}[\nabla y(x)-\pi(\nabla y(x))] w \cdot F_{\lambda} w \mathrm{~d} x .
\end{aligned}
$$


We obtain from the Cauchy-Schwarz inequality and the above inequality (3.7) that

$$
\left|\int_{\Omega}[\nabla y(x)-\pi(\nabla y(x))] w \cdot F_{\lambda} w \mathrm{~d} x\right| \leq C \mathcal{E}(y)^{1 / 2} .
$$

So, it follows from (3.10) that for all $w \in \mathbb{R}^{3}$ with $|w|=1$

$$
\rho_{1}(y ; w)=\sum_{k=1}^{4} \tau_{k}(y)\left(\left|F_{\lambda} w\right|^{2}-\left|U_{k} w\right|^{2}\right) \leq C \mathcal{E}(y)^{1 / 2} .
$$

The result (3.8) then follows from the above inequality (3.11) and (3.3).

Next, we obtain similar estimates for the cofactor. We have from (3.1) and (3.6) that for any $w \in \mathbb{R}^{3},|w|=1$,

$$
\begin{aligned}
\rho_{2}(y ; w)= & \sum_{k=1}^{4} \tau_{k}(y)\left[\left|\left(\operatorname{Cof} F_{\lambda}\right) w\right|^{2}-\left|\left(\operatorname{Cof} U_{k}\right) w\right|^{2}\right] \\
= & \frac{1}{\operatorname{meas} \Omega} \int_{\Omega}\left[\left|\left(\operatorname{Cof} F_{\lambda}\right) w\right|^{2}-|(\operatorname{Cof} \pi(\nabla y(x))) w|^{2}\right] \mathrm{d} x \\
= & -\frac{1}{\operatorname{meas} \Omega} \int_{\Omega}\left|\left[\operatorname{Cof} F_{\lambda}-\operatorname{Cof} \pi(\nabla y(x))\right] w\right|^{2} \mathrm{~d} x \\
& +\frac{2}{\operatorname{meas} \Omega} \int_{\Omega}[\operatorname{Cof} \nabla y(x)-\operatorname{Cof} \pi(\nabla y(x))] w \cdot\left(\operatorname{Cof} F_{\lambda}\right) w \mathrm{~d} x \\
\leq & \frac{2}{\operatorname{meas} \Omega} \int_{\Omega}[\operatorname{Cof} \nabla y(x)-\operatorname{Cof} \pi(\nabla y(x))] w \cdot\left(\operatorname{Cof} F_{\lambda}\right) w \mathrm{~d} x .
\end{aligned}
$$

Letting $F(x)=\nabla y(x)$ for $x \in \Omega$, we have that $F(x)=\left(F_{k l}(x)\right) \in \mathrm{L}^{2}\left(\Omega ; \mathbb{R}^{3 \times 3}\right)$. Now $\pi(\nabla y(x)) \in \mathcal{U}$ for all $x \in \Omega$, so if we set $P(x)=\pi(\nabla y(x))$ for $x \in \Omega$ we have that $P(x)=\left(P_{k l}(x)\right)$ is uniformly bounded in $\mathrm{L}^{\infty}\left(\Omega ; \mathbb{R}^{3 \times 3}\right)$ for all $y \in \mathcal{A}$. We have for any $k, l, p, q \in\{1,2,3\}$ that

$$
F_{k l} F_{p q}-P_{k l} P_{p q}=\left(F_{k l}-P_{k l}\right) P_{p q}+P_{k l}\left(F_{p q}-P_{p q}\right)+\left(F_{k l}-P_{k l}\right)\left(F_{p q}-P_{p q}\right) .
$$

Hence, we have by the Cauchy-Schwarz inequality and (3.7) that

$$
\int_{\Omega}|[\operatorname{Cof} \nabla y(x)-\operatorname{Cof} \pi(\nabla y(x))] w| \mathrm{d} x \leq C\left[\mathcal{E}(y)^{1 / 2}+\mathcal{E}(y)\right] .
$$

Thus, we have from (3.12) and (3.13) that

$$
\begin{aligned}
\rho_{2}(y ; w) & =\sum_{k=1}^{4} \tau_{k}(y)\left[\left|\left(\operatorname{Cof} F_{\lambda}\right) w\right|^{2}-\left|\left(\operatorname{Cof} U_{k}\right) w\right|^{2}\right] \\
& \leq C\left[\mathcal{E}(y)^{1 / 2}+\mathcal{E}(y)\right] \quad \text { for all } w \in \mathbb{R}^{3},|w|=1
\end{aligned}
$$

The result (3.9) then follows from the above inequality (3.14) and (3.5).

We will use Lemma 3.1 to establish the following inequality for all material parameters not satisfying certain identities:

$$
\tau_{k}(y) \leq C\left[\mathcal{E}(y)^{1 / 2}+\mathcal{E}(y)\right] \quad \text { for all } k \in\{1, \ldots, 4\} \backslash\{i, j\} \text { and all } y \in \mathcal{A}
$$


We will also give conditions on the material parameters $\theta_{i}, \delta_{i}$, and $\eta_{i}$ under which this inequality cannot be established. This, in turn, will lead to uniqueness or nonuniqueness of the Young measures associated with energy minimizing sequences of deformations, which we discuss in the next section.

Theorem 3.1. Assume that $\phi$ satisfies (2.1), (2.2), and (2.4), $F_{\lambda}$ is defined as in $(2.12)$ with $\lambda \in(0,1)$, and $\mathcal{A}$ is defined by (2.14).

Case 1A: Suppose $(i, j)$ in the definition of $F_{\lambda}$ determines either of the compound twins with $n=n_{1}$. Then (3.15) holds for all the parameters $\theta_{i}$, except those that satisfy

$$
\theta_{2}^{2}\left(\theta_{3}^{2}+\theta_{4}^{2}\right)=\left(\theta_{1} \theta_{3}-\theta_{4}^{2}\right)^{2}
$$

in which case (3.15) does not hold for $\lambda=1 / 2$.

Case 1B: Suppose $(i, j)$ in the definition of $F_{\lambda}$ determines either of the compound twins with $n=n_{2}$. Then (3.15) holds for all the parameters $\theta_{i}$, except those that satisfy

$$
\theta_{2}^{2}=\theta_{1}^{2}+\theta_{4}^{2}
$$

in which case (3.15) does not hold for $\lambda=1 / 2$.

Case 1C: Suppose $(i, j)$ and $n$ in the definition of $F_{\lambda}$ determine any of the remaining type $I$ or type II twins. Then (3.15) holds for all the parameters $\theta_{i}$.

Case 2A: Suppose $(i, j)$ in the definition of $F_{\lambda}$ determines any of the four compound twins with $n=n_{1}$. Then (3.15) holds for all the parameters $\delta_{i}$.

Case 2B: Suppose $(i, j)$ in the definition of $F_{\lambda}$ determines any other twin than those in Case $2 A$ above. Then (3.15) does not hold for any choice of the parameters $\delta_{i}$.

Case 3A: Suppose $(i, j)$ in the definition of $F_{\lambda}$ determines either of the compound twins with $n=n_{1}$. Then (3.15) holds for all the parameters $\eta_{i}$, except those that satisfy

$$
2 \eta_{1} \eta_{3}=\eta_{4}^{2}
$$

in which case (3.15) does not hold for $\lambda=1 / 2$.

Case 3B: Suppose $(i, j)$ in the definition of $F_{\lambda}$ determines any other twin than those in Case $3 A$ above. Then (3.15) holds for any choice of the parameters $\eta_{i}$.

Proof. Case 1A. Assume that $(i, j)=(1,2)$ and $n=e_{1}$. Since this is a compound twin, it follows from Lemma 2.1 that $U_{2}^{-1} a$ is parallel to $e_{3}$. Let $s, t \in \mathbb{R}$ be such that $w=(s, t, 0)^{T}$ has unit length. Then using Lemma 3.1 we have

$$
\begin{aligned}
\rho_{2}(y ; w) & =\left(s^{2}-t^{2}\right)\left(\theta_{2}^{2}\left(\theta_{3}^{2}+\theta_{4}^{2}\right)-\left(\theta_{1} \theta_{3}-\theta_{4}^{2}\right)^{2}\right)\left[\tau_{3}(y)+\tau_{4}(y)\right] \\
& \leq C\left[\mathcal{E}(y)^{1 / 2}+\mathcal{E}(y)\right]
\end{aligned}
$$

If (3.16) does not hold, then we can choose $s$ and $t$ such that

$$
\left(s^{2}-t^{2}\right)\left(\theta_{2}^{2}\left(\theta_{3}^{2}+\theta_{4}^{2}\right)-\left(\theta_{1} \theta_{3}-\theta_{4}^{2}\right)^{2}\right)>0 .
$$

Therefore,

$$
\tau_{3}(y)+\tau_{4}(y) \leq C\left[\mathcal{E}(y)^{1 / 2}+\mathcal{E}(y)\right] \quad \text { for } \theta_{2}^{2}\left(\theta_{3}^{2}+\theta_{4}^{2}\right) \neq\left(\theta_{1} \theta_{3}-\theta_{4}^{2}\right)^{2}
$$

Let us now assume that (3.16) holds. We show that if $\lambda=1 / 2$, then we can construct a sequence $\left\{y_{n}\right\} \subset \mathcal{A}$ of deformations whose energy converges to 0 , but the volume fractions $\tau_{3}\left(y_{n}\right)$ and $\tau_{4}\left(y_{n}\right)$ converge to $1 / 2$. 
Using Lemma 2.1 and the transformation matrices (2.6), we obtain after a series of calculations that

$$
a=\frac{2 \hat{\sigma}}{\theta_{3}^{2}+\theta_{4}^{2}}\left(\begin{array}{c}
-\theta_{4} \\
0 \\
\theta_{3}
\end{array}\right)
$$

where

$$
\hat{\sigma}=\theta_{4}\left(\theta_{1}+\theta_{3}\right)>0
$$

Using the fact that

$$
F_{\lambda}=U_{2}+\lambda a \otimes e_{1}
$$

we obtain

$$
F_{\lambda}^{T} F_{\lambda}=\left(\begin{array}{ccc}
\sigma(\lambda) & 0 & (2 \lambda-1) \hat{\sigma} \\
0 & \theta_{2}^{2} & 0 \\
(2 \lambda-1) \hat{\sigma} & 0 & \theta_{3}^{2}+\theta_{4}^{2}
\end{array}\right)
$$

where

$$
\sigma(\lambda)=\theta_{1}^{2}+\theta_{4}^{2}+4 \lambda(\lambda-1) \frac{\hat{\sigma}^{2}}{\theta_{3}^{2}+\theta_{4}^{2}} .
$$

Recall that $R_{5}^{T} U_{1} R_{5}=U_{3}$ and $R_{5}^{T} U_{2} R_{5}=U_{4}$, so that

$$
Q U_{1}-U_{2}=a \otimes e_{1}
$$

is equivalent to

$$
\tilde{Q} U_{3}-U_{4}=-R_{5} a \otimes e_{2}
$$

with $\tilde{Q}=R_{5}^{T} Q R_{5}$. Setting

$$
G_{\lambda}=\lambda \tilde{Q} U_{3}+(1-\lambda) U_{4}=R_{5}^{T} F_{\lambda} R_{5},
$$

we have

$$
G_{\lambda}^{T} G_{\lambda}=\left(\begin{array}{ccc}
\theta_{2}^{2} & 0 & 0 \\
0 & \sigma(\lambda) & (2 \lambda-1) \hat{\sigma} \\
0 & (2 \lambda-1) \hat{\sigma} & \theta_{3}^{2}+\theta_{4}^{2}
\end{array}\right)
$$

and we conclude that

$$
G_{\lambda}^{T} G_{\lambda}=F_{\lambda}^{T} F_{\lambda} \quad \text { if and only if } \quad \lambda=1 / 2 \text { and } \sigma(\lambda)=\theta_{2}^{2} .
$$

However, it is easy to check that $\sigma(1 / 2)=\theta_{2}^{2}$ is equivalent to (3.16). Therefore, if (3.16) holds, then $F_{1 / 2}=$ $\bar{Q} G_{1 / 2}$ for some $\bar{Q} \in \mathrm{SO}(3)$, and hence we can construct a sequence of deformations $\left\{y_{n}\right\} \subset \mathcal{A}$ with $\mathcal{E}\left(y_{n}\right) \rightarrow 0$ such that the volume fractions $\tau_{3}\left(y_{n}\right) \rightarrow 1 / 2$ and $\tau_{4}\left(y_{n}\right) \rightarrow 1 / 2$ [12,28]. This proves that (3.15) cannot be proven if $\lambda=1 / 2$ and (3.16) holds.

The proof for case $(i, j)=(3,4)$ and $n=e_{2}$ follows by symmetry since $R_{5}^{T} U_{1} R_{5}=U_{3}$ and $R_{5}^{T} U_{2} R_{5}=U_{4}$. 
Case 1B. Consider the case $(i, j)=(1,2)$ and $n=e_{3}$. Let $s, t \in \mathbb{R}$ be such that $w=(s, t, 0)^{T}$ has unit length. Then we have

$$
\begin{aligned}
\rho_{1}(y ; w) & =\left(s^{2}-t^{2}\right)\left(\theta_{1}^{2}+\theta_{4}^{2}-\theta_{2}^{2}\right)\left[\tau_{3}(y)+\tau_{4}(y)\right] \\
& \leq C \mathcal{E}(y)^{1 / 2}
\end{aligned}
$$

which leads to

$$
\tau_{3}(y)+\tau_{4}(y) \leq C \mathcal{E}(y)^{1 / 2} \quad \text { for } \theta_{2}^{2} \neq \theta_{1}^{2}+\theta_{4}^{2} .
$$

Proceeding now as in the previous part, we can define $G_{\lambda}=R_{5}^{T} F_{\lambda} R_{5}$ which corresponds to the compound twin with $(i, j)=(3,4)$ and $n=e_{3}$, and again we conclude that

$$
G_{\lambda}^{T} G_{\lambda}=F_{\lambda}^{T} F_{\lambda} \quad \text { if and only if } \quad \lambda=1 / 2 \text { and (3.17) holds, }
$$

leading again to a sequence of deformations $\left\{y_{n}\right\} \subset \mathcal{A}$ with $\mathcal{E}\left(y_{n}\right) \rightarrow 0$ and the volume fractions $\tau_{3}\left(y_{n}\right) \rightarrow 1 / 2$ and $\tau_{4}\left(y_{n}\right) \rightarrow 1 / 2$. $U_{4}$.

The proof for case $(i, j)=(3,4)$ and $n=e_{3}$ again follows by symmetry since $R_{5}^{T} U_{1} R_{5}=U_{3}$ and $R_{5}^{T} U_{2} R_{5}=$

Case 1C. Consider first the case $(i, j)=(1,3)$ and $n=e_{1}-e_{2}$. Let $s, t \in \mathbb{R}$ be such that $w=(s, s, t)^{T}$ has unit length and $s t>0$. Then we have since $\hat{\sigma}>0$ that

$$
\begin{aligned}
\rho_{1}(y ; w) & =4 s t \hat{\sigma}\left[\tau_{2}(y)+\tau_{4}(y)\right] \\
& \leq C \mathcal{E}(y)^{1 / 2}
\end{aligned}
$$

leading to

$$
\tau_{2}(y)+\tau_{4}(y) \leq C \mathcal{E}(y)^{1 / 2} .
$$

Next let $(i, j)=(1,3)$ and $n=\left(-\varepsilon_{1},-\varepsilon_{1}, \sqrt{1-2 \varepsilon_{1}^{2}}\right)$. Since this is a type II twin, it follows from Lemma 2.1 that $U_{3}^{-1} a$ is parallel to $e_{1}-e_{2}$. Let $s, t \in \mathbb{R}$ be such that $w=(s, s, t)^{T}$ has unit length and $s t<0$. Then we have

$$
\begin{aligned}
\rho_{2}(y ; w) & =-4 s t \hat{\sigma} \theta_{2}^{2}\left[\tau_{2}(y)+\tau_{4}(y)\right] \\
& \leq C\left[\mathcal{E}(y)^{1 / 2}+\mathcal{E}(y)\right]
\end{aligned}
$$

leading to

$$
\tau_{2}(y)+\tau_{4}(y) \leq C\left[\mathcal{E}(y)^{1 / 2}+\mathcal{E}(y)\right]
$$

The proof for cases $(i, j)=(1,4),(i, j)=(2,3)$, and $(i, j)=(2,4)$ follows from symmetry since $R_{2}^{T} U_{1} R_{2}=U_{1}$ and $R_{2}^{T} U_{3} R_{2}=U_{4}, R_{1}^{T} U_{1} R_{1}=U_{2}$ and $R_{1}^{T} U_{3} R_{1}=U_{3}$, and $R_{3}^{T} U_{1} R_{3}=U_{2}$ and $R_{3}^{T} U_{3} R_{3}=U_{4}$.

Case 2A. Assume first that $(i, j)=(1,2)$ and $n=e_{2}$. We evaluate $\rho_{1}\left(y ; e_{1}\right)$ to get

$$
\begin{aligned}
\rho_{1}\left(y ; e_{1}\right) & =\left[\tau_{3}(y)+\tau_{4}(y)\right]\left(\delta_{1}^{2}-\delta_{2}^{2}\right) \\
& \leq C \mathcal{E}(y)^{1 / 2}
\end{aligned}
$$


leading to

$$
\tau_{3}(y)+\tau_{4}(y) \leq C \mathcal{E}(y)^{1 / 2}
$$

since we assumed $\delta_{1}>\delta_{2}>0$.

The proof for case $(i, j)=(3,4)$ and $n=e_{1}$ follows by symmetry since $R_{4}^{T} U_{1} R_{4}=U_{3}$ and $R_{4}^{T} U_{2} R_{4}=U_{4}$.

Assume next that $(i, j)=(1,3)$ and $n=e_{1}-e_{2}$. Let $w=\left(e_{1}+e_{2}\right) / \sqrt{2}$. Then

$$
\begin{aligned}
\rho_{1}(y ; w) & =\left[\tau_{2}(y)+\tau_{4}(y)\right] 2 \delta_{4}\left(\delta_{1}+\delta_{2}\right) \\
& \leq C \mathcal{E}(y)^{1 / 2}
\end{aligned}
$$

leading to

$$
\tau_{2}(y)+\tau_{4}(y) \leq C \mathcal{E}(y)^{1 / 2}
$$

The proof for case $(i, j)=(2,4)$ and $n=e_{1}+e_{2}$ follows by symmetry since $R_{1}^{T} U_{1} R_{1}=U_{2}$ and $R_{1}^{T} U_{3} R_{1}=U_{4}$.

Case 2B. In this case, the energy wells given by the transformation matrices in (2.7) are essentially twodimensional, so the results given by Bhattacharya and Dolzmann in Example 7.3 in [5] give a proof of the assertion in Case 2B. For completeness, we give a modified version of their proof here.

We set $\delta=\operatorname{det} U_{i}$ and consider the set $\mathcal{C}$ of symmetric positive definite matrices with determinant equal to $\delta^{2}$, of the form

$$
C=\left(\begin{array}{ccc}
C_{11} & C_{12} & 0 \\
C_{12} & C_{22} & 0 \\
0 & 0 & \delta_{3}^{2}
\end{array}\right)
$$

Then there is a one-to-one correspondence between $\mathcal{C}$ and $\mathbb{R}^{2}$ given by

$$
C \mapsto\left(C_{11}-C_{22}, 2 C_{12}\right),
$$

and we shall implicitly assume this correspondence in what follows. Under this map,

$$
\begin{aligned}
U_{1}^{2} \mapsto\left(\delta_{1}^{2}-\delta_{2}^{2}, 2 \delta_{4}\left(\delta_{1}+\delta_{2}\right)\right), & U_{2}^{2} \mapsto\left(\delta_{1}^{2}-\delta_{2}^{2},-2 \delta_{4}\left(\delta_{1}+\delta_{2}\right)\right), \\
U_{3}^{2} \mapsto\left(\delta_{2}^{2}-\delta_{1}^{2}, 2 \delta_{4}\left(\delta_{1}+\delta_{2}\right)\right), & U_{4}^{2} \mapsto\left(\delta_{2}^{2}-\delta_{1}^{2},-2 \delta_{4}\left(\delta_{1}+\delta_{2}\right)\right) .
\end{aligned}
$$

Similarly as for the energy wells $\mathcal{U}_{i}$, we define rank-one connections between sets $\mathcal{V}_{1}=\mathrm{SO}(3) V_{1}$ and $\mathcal{V}_{2}=\operatorname{SO}(3) V_{2}$ where $V_{1}, V_{2} \in \mathbb{R}^{3 \times 3}$. We say that $\mathcal{V}_{1}$ and $\mathcal{V}_{2}$ are rank-one connected if there exist $Q \in \mathrm{SO}(3), a \in \mathbb{R}^{3}, a \neq 0$, and $n \in \mathbb{R}^{3}, n \neq 0$, such that

$$
Q V_{2}=V_{1}+a \otimes n
$$

Note that if $\operatorname{det} V_{1}=\operatorname{det} V_{2}$, then $V_{1}^{-1} a \cdot n=0$ and $\operatorname{det}\left(\lambda Q V_{2}+(1-\lambda) V_{1}\right)=\operatorname{det} V_{1}$. Note also that if $V_{1}$ and $V_{2}$ are symmetric positive definite, we can identify $\mathcal{V}_{1}$ with $V_{1}^{2}$ and $\mathcal{V}_{2}$ with $V_{2}^{2}$ and, abusing the language slightly, talk about rank-one connections between $V_{1}^{2}$ and $V_{2}^{2}$, and in particular, between elements of $\mathcal{C}$, or, correspondingly, between points in $\mathbb{R}^{2}$ under the identification (3.19).

Consider now two distinct symmetric positive definite matrices $A, B$ such that $A^{2}, B^{2} \in \mathcal{C}$. It then follows from Lemma 5 in [28] that there exist two rank-one connections

$$
Q B=A+a \otimes n
$$




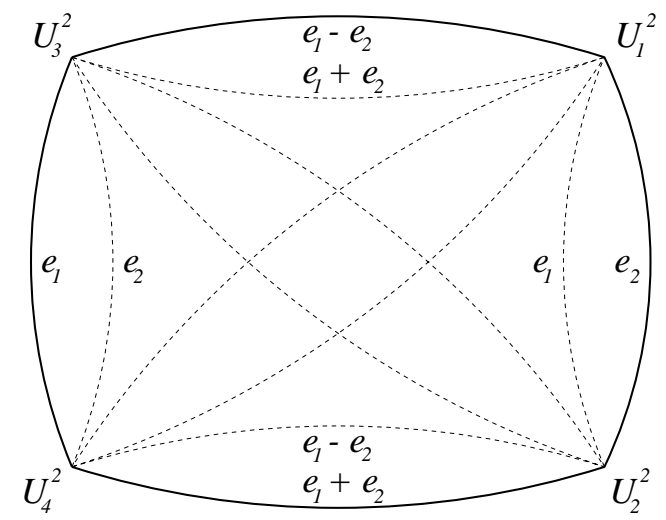

Figure 1. The rank-one connections in Case 2.

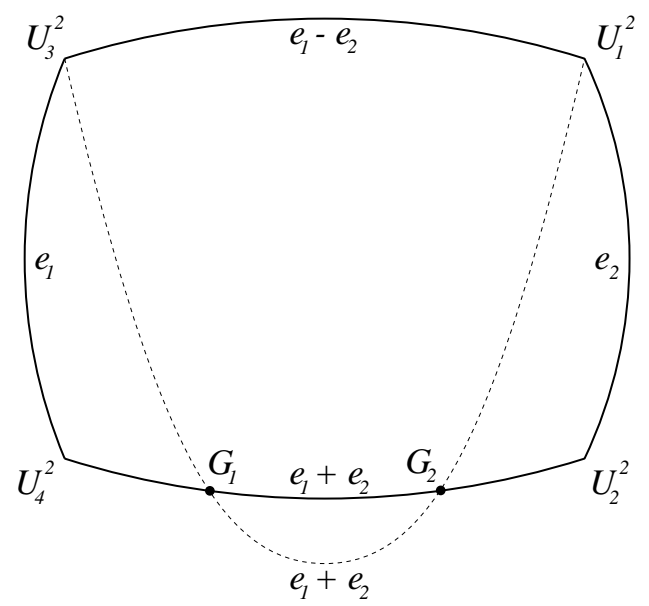

FIgURE 2. The curves of rank-one connections for compound twins with $n=n_{2}$ must lie in the interior of $\mathcal{D}$.

such that $n \cdot e_{3}=a \cdot e_{3}=0$, and $Q=R\left(\alpha, e_{3}\right)$ for some $\alpha \in \mathbb{R}$. This implies that $A^{-1} a \cdot n=0$, and we can write

$$
Q B=A\left(I+s n^{\perp} \otimes n\right)
$$

for some $s \in \mathbb{R}$, where $n^{\perp}=R\left(\pi / 2, e_{3}\right) n$. Thus,

$$
B^{2}=A^{2}+s\left(A^{2} n^{\perp} \otimes n+n \otimes A^{2} n^{\perp}\right)+s^{2}\left|A n^{\perp}\right|^{2} n \otimes n
$$

and we conclude that two matrices in $\mathcal{C}$ are rank-one connected if and only if they lie on a quadratic curve parametrized by $(3.20)$ for $s \in \mathbb{R}$.

We define the vertex of a parabola to be its point of maximum curvature. We also define its axis to be the half-line interior to the parabola that extends from the vertex to infinity. Writing $n=|n|(\cos \theta, \sin \theta, 0)$ and letting $\tilde{A}=A^{2}$ and $\tilde{B}=B^{2}$, we have

$$
\begin{gathered}
\tilde{B}_{11}-\tilde{B}_{22}=\tilde{A}_{11}-\tilde{A}_{22}+s|n|^{2}\left(2 \tilde{A}_{12}-\left(\tilde{A}_{11}+\tilde{A}_{22}\right) \sin 2 \theta\right)+s^{2}|n|^{2}\left|A n^{\perp}\right|^{2} \cos 2 \theta \\
2 \tilde{B}_{12}=2 \tilde{A}_{12}+2 s|n|^{2}\left(\tilde{A}_{22} \cos ^{2} \theta-\tilde{A}_{11} \sin ^{2} \theta\right)+s^{2}|n|^{2}\left|A n^{\perp}\right|^{2} \sin 2 \theta
\end{gathered}
$$




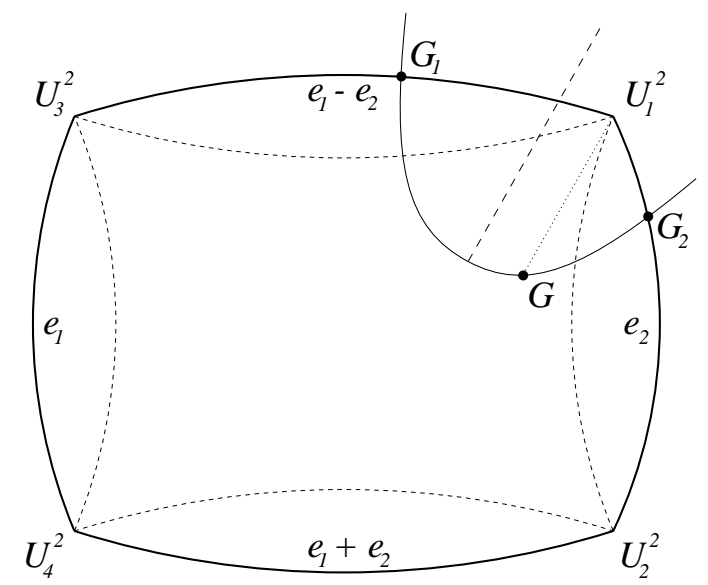

Figure 3. There is a curve of rank-one connections through any $G \in \mathcal{D}$ that intersects two of the parabolic curves that bound $\mathcal{D}$.

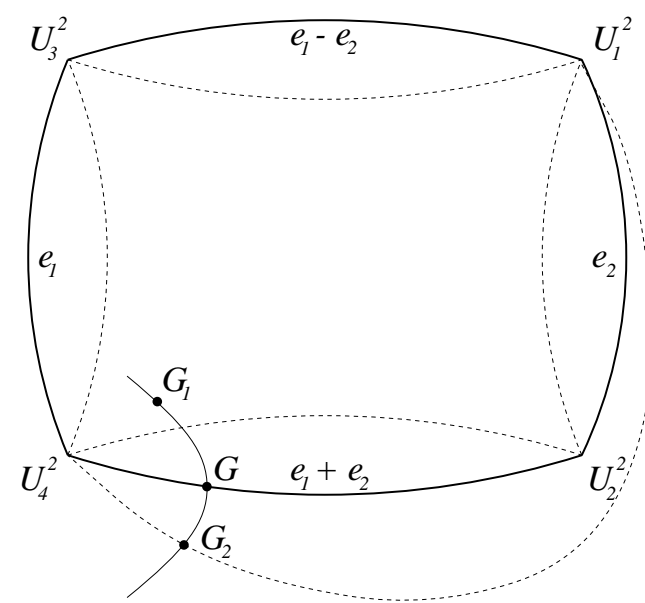

FiguRE 4. The curve of rank-one connections for domain interfaces must lie in the interior of $\mathcal{D}$.

where $\left|A n^{\perp}\right|^{2}>0$. It can be seen that in the $\left(C_{11}-C_{22}, 2 C_{12}\right)$-plane this curve is a parabola with axis in the direction $(\cos 2 \theta, \sin 2 \theta)$. We note that this curve cannot cross any point $G \in \mathcal{C}$ twice since otherwise $G$ would be rank-one connected to itself. We can also see that this curve does not degenerate since

$$
\left(2 \tilde{A}_{12}-\left(\tilde{A}_{11}+\tilde{A}_{22}\right) \sin 2 \theta\right) \sin 2 \theta \neq 2\left(\tilde{A}_{22} \cos ^{2} \theta-\tilde{A}_{11} \sin ^{2} \theta\right) \cos 2 \theta,
$$

for any positive definite matrix $\tilde{A} \in \mathcal{C}$. Therefore, the four rank-one connections from Case $2 \mathrm{~A}$ corresponding to compound twins with $n=n_{1}$ (see Lemma 2.3 for definition of $n_{1}$ ) determine a closed curve through the $U_{i}^{2}, i=1, \ldots, 4$, in the $\left(C_{11}-C_{22}, 2 C_{12}\right)$-plane consisting of four parabolic segments bulging out of the domain $\mathcal{D}$ they are bounding (see Fig. 1 ).

The other four compound twins with $n=n_{2}$ determine parabolas through the corresponding $U_{i}^{2}$ with axes pointing in the opposite direction to the axis of the curve of rank-one connections corresponding to its compound twin system for $n=n_{1}$. We claim that their vertices (thus the whole segments joining $U_{i}^{2}$ to $U_{j}^{2}$ for the corresponding $i$ and $j$ ) lie in $\mathcal{D}$. Assume this is not so, that is, assume that there exists a compound twin determined by $U_{i}$ and $U_{j}$ with $n=n_{2}$ such that the vertex of the corresponding parabola does not lie in $\mathcal{D}$. We visualize this example in Figure 2 for the parabolic curve of rank-one connections connecting $U_{1}^{2}$ and $U_{3}^{2}$ with 
$n_{2}=e_{1}+e_{2}$ corresponding by Lemma 2.1 to

$$
\left(U_{3}+\lambda a_{2} \otimes n_{2}\right)^{T}\left(U_{3}+\lambda a_{2} \otimes n_{2}\right) \in \mathcal{C}
$$

where

$$
n_{2}=e_{1}+e_{2}, \quad a_{2}=2 \frac{U_{3}^{-1} n_{2}}{\left|U_{3}^{-1} n_{2}\right|^{2}}-U_{3} n_{2}
$$

In this case, we suppose that the parabola intersects the curve of rank-one connections connecting $U_{2}^{2}$ and $U_{4}^{2}$ with $n_{1}=e_{1}+e_{2}$ at a point

$$
G_{1}=\left(U_{3}+\lambda a_{2} \otimes n_{2}\right)^{T}\left(U_{3}+\lambda a_{2} \otimes n_{2}\right) \in \mathcal{C}
$$

for $0<\lambda<1$. For an admissible space of deformations

$$
\mathcal{A}=\left\{y \in W^{\phi}: y(x)=y_{0}(x) \text { for all } x \in \partial \Omega\right\}
$$

where

$$
y_{0}(x)=\sqrt{G_{1}} x \quad \text { for all } x \in \Omega,
$$

we can then construct a sequence of laminated deformations $\left\{y_{n}\right\} \subset \mathcal{A}$ with $\mathcal{E}\left(y_{n}\right) \rightarrow 0$ such that the volume fractions $\tau_{1}\left(y_{n}\right) \rightarrow \lambda \neq 0$ and $\tau_{3}\left(y_{n}\right) \rightarrow 1-\lambda \neq 0$. This contradicts the result in Case $2 \mathrm{~A}$ that since $G_{1}$ lies on the curve of rank-one connections connecting $U_{2}^{2}$ and $U_{4}^{2}$ with $n_{1}=e_{1}+e_{2}$ we must have that $\tau_{1}\left(y_{n}\right) \rightarrow 0$ and $\tau_{3}\left(y_{n}\right) \rightarrow 0$. for

Next, we let $G \in \mathcal{C}$ be a point in the interior of $\mathcal{D}$, and we then construct a sequence of deformations $\left\{y_{n}\right\} \subset \mathcal{A}$

$$
\mathcal{A}=\left\{y \in W^{\phi}: y(x)=y_{0}(x) \text { for all } x \in \partial \Omega\right\}
$$

where

$$
y_{0}(x)=\sqrt{G} x \quad \text { for all } x \in \Omega,
$$

such that $\mathcal{E}\left(y_{n}\right) \rightarrow 0$ and $\tau_{k}\left(y_{n}\right) \nrightarrow 0$ for each $k \in\{1, \ldots, 4\}$. This result then provides a proof of Case $2 \mathrm{~B}$ for the four compound twin families with $n=n_{2}$. We define $F=\sqrt{G}$ and let

$$
F_{\lambda}=F\left(I+\lambda v^{\perp} \otimes v\right)
$$

for some $v \in \mathbb{R}^{3}, v \neq 0$. This determines a rank-one curve passing through $G$ that intersects the boundary of $\mathcal{D}$ at two points $G_{1}$ and $G_{2}$. If we choose $v$ as in Figure 3 such that the axis of the parabola is in the direction $U_{i}^{2}-G$ for some $i=1, \ldots, 4$, then we know that the two intersections $G_{1}$ and $G_{2}$ lie on different parabolic segments of the boundary of $\mathcal{D}$. We can now construct a sequence of deformations $\left\{y_{n}\right\} \subset \mathcal{A}$ such that $\mathcal{E}\left(y_{n}\right) \rightarrow 0$ and $\tau_{k}\left(y_{n}\right) \nrightarrow 0$ for those $k$ for which $U_{k}^{2}$ participates in the rank-one connections for the parabolic segments on the boundary of $\mathcal{D}$ corresponding to $G_{1}$ and $G_{2}$. By rotating $v$ we see that we can construct a sequence $\left\{y_{n}\right\} \subset \mathcal{A}$ such that $\mathcal{E}\left(y_{n}\right) \rightarrow 0$ and $\tau_{k}\left(y_{n}\right) \nrightarrow 0$ for every $k \in\{1, \ldots, 4\}$.

Finally, if we show that the parabolic segments of rank-one connections extending from $U_{1}^{2}$ to $U_{4}^{2}$ and from $U_{2}^{2}$ to $U_{3}^{2}$ also lie in $\mathcal{D}$, the proof will be complete. However, if this were not so, there would exist a curve of rank-one connections passing through a point $G$ on a parabolic segment of the boundary of $\mathcal{D}$, a point $G_{1}$ in the interior of $\mathcal{D}$, and a point $G_{2}$ on one of the parabolic segments of rank-one connections extending from $U_{1}^{2}$ 
to $U_{4}^{2}$ or from $U_{2}^{2}$ to $U_{3}^{2}$ (see Figure 4 ). However, we can see as above that this violates the result in Case $2 \mathrm{~A}$ for the boundary segment. have

Case 3A. Assume that $(i, j)=(1,3)$ and $n=e_{3}$. Let $w=(s, t, 0)^{T}$ for $s, t \in \mathbb{R}$ be of unit length. Then we

$$
\begin{aligned}
\rho_{1}(y ; w) & =4 s t\left(2 \eta_{1} \eta_{3}-\eta_{4}^{2}\right)\left[\tau_{2}(y)+\tau_{4}(y)\right] \\
& \leq C \mathcal{E}(y)^{1 / 2} .
\end{aligned}
$$

Choosing the sign of $s t$ to be the same as that of $2 \eta_{1} \eta_{3}-\eta_{4}^{2}$, we conclude that

$$
\tau_{2}(y)+\tau_{4}(y) \leq C \mathcal{E}(y)^{1 / 2} \quad \text { for } 2 \eta_{1} \eta_{3} \neq \eta_{4}^{2} .
$$

Assume now that $2 \eta_{1} \eta_{3}=\eta_{4}^{2}$. After a series of calculations, we then find that

$$
a=\frac{2 \hat{\sigma}}{\left(\eta_{1}+\eta_{3}\right)^{2}}\left(\begin{array}{c}
\eta_{1}-\eta_{3} \\
-\left(\eta_{1}-\eta_{3}\right) \\
-2 \eta_{4}
\end{array}\right)
$$

and

$$
F_{\lambda}^{T} F_{\lambda}=\left(\begin{array}{ccc}
\left(\eta_{1}+\eta_{3}\right)^{2} & 0 & (2 \lambda-1) \hat{\sigma} \\
0 & \left(\eta_{1}+\eta_{3}\right)^{2} & -(2 \lambda-1) \hat{\sigma} \\
(2 \lambda-1) \hat{\sigma} & -(2 \lambda-1) \hat{\sigma} & \sigma(\lambda)
\end{array}\right)
$$

where

$$
\hat{\sigma}=\eta_{4}\left(\eta_{1}+\eta_{2}-\eta_{3}\right)>0
$$

and

$$
\sigma(\lambda)=\eta_{2}^{2}+2 \eta_{4}^{2}+8 \lambda(\lambda-1) \frac{\hat{\sigma}^{2}}{\left(\eta_{1}+\eta_{3}\right)^{2}}
$$

Recalling that $R_{2}^{T} U_{1} R_{2}=U_{2}$ and $R_{2}^{T} U_{3} R_{2}=U_{4}$, we define $G_{\lambda}=R_{2}^{T} F_{\lambda} R_{2}$ as in the proof for Case 1 and conclude that $G_{1 / 2}^{T} G_{1 / 2}=F_{1 / 2}^{T} F_{1 / 2}$ when (3.18) holds. Therefore we can construct a sequence of deformations $\left\{y_{n}\right\} \subset \mathcal{A}$ with $\mathcal{E}\left(y_{n}\right) \rightarrow 0$ and the volume fractions $\tau_{2}\left(y_{n}\right) \rightarrow 1 / 2$ and $\tau_{4}\left(y_{n}\right) \rightarrow 1 / 2$.

The proof for case $(i, j)=(2,4)$ and $n=e_{3}$ follows by symmetry since $R_{2}^{T} U_{1} R_{2}=U_{2}$ and $R_{2}^{T} U_{3} R_{2}=U_{4}$.

Case 3B. Consider first the case $(i, j)=(1,3)$ and $n=e_{1}-e_{2}$. Since this is a compound twin, we have that $U_{3}^{-1} a$ is parallel to $e_{3}$. Let $s, t \in \mathbb{R}$ be such that $w=(s, t, 0)^{T}$ has unit length and $s t<0$. We then have that

$$
\begin{aligned}
\rho_{2}(y ; w) & =-4 s t\left(2\left(\eta_{1} \eta_{2}-\eta_{4}^{2}\right)\left(\eta_{2} \eta_{3}+\eta_{4}^{2}\right)+\eta_{4}^{2}\left(\eta_{1}+\eta_{3}\right)^{2}\right)\left[\tau_{2}(y)+\tau_{4}(y)\right] \\
& \leq C\left[\mathcal{E}(y)^{1 / 2}+\mathcal{E}(y)\right]
\end{aligned}
$$

Since we have $\eta_{1} \eta_{2}-\eta_{4}^{2}>0$, it follows that

$$
\tau_{2}(y)+\tau_{4}(y) \leq C\left[\mathcal{E}(y)^{1 / 2}+\mathcal{E}(y)\right]
$$

The proof for case $(i, j)=(2,4)$ and $n=e_{1}+e_{2}$ follows by symmetry since $R_{2}^{T} U_{1} R_{2}=U_{2}$ and $R_{2}^{T} U_{3} R_{2}=U_{4}$. 
Consider the case $(i, j)=(1,2)$ and the type I interface $n=e_{2}$. Let $s, t \in \mathbb{R}$ be such that $w=(s, 0, t)^{T}$ has unit length and $s t>0$. We then have since $\hat{\sigma}>0$ that

$$
\begin{aligned}
\rho_{1}(y ; w) & =4 s t \hat{\sigma}\left[\tau_{2}(y)+\tau_{4}(y)\right] \\
& \leq C \mathcal{E}(y)^{1 / 2},
\end{aligned}
$$

leading to

$$
\tau_{3}(y)+\tau_{4}(y) \leq C \mathcal{E}(y)^{1 / 2} .
$$

Consider next the case $(i, j)=(1,2)$ and the type II interface $n=\left(\varepsilon_{2}, 0,-\sqrt{1-\varepsilon_{2}^{2}}\right)$. Since this is a type II twin, we have that $U_{2}^{-1} a$ is parallel to $e_{2}$. Let $s, t \in \mathbb{R}$ be such that $w=(s, 0, t)^{T}$ has unit length and $s t<0$. We then have that

$$
\begin{aligned}
\rho_{2}(y ; w) & =-4 s t \hat{\sigma}\left(\eta_{1}+\eta_{3}\right)^{2}\left[\tau_{3}(y)+\tau_{4}(y)\right] \\
& \leq C\left[\mathcal{E}(y)^{1 / 2}+\mathcal{E}(y)\right]
\end{aligned}
$$

leading to

$$
\tau_{3}(y)+\tau_{4}(y) \leq C\left[\mathcal{E}(y)^{1 / 2}+\mathcal{E}(y)\right] .
$$

The proof for the interfaces for cases $(i, j)=(1,4),(i, j)=(2,3)$, and $(i, j)=(3,4)$ follows by symmetry since $R_{4}^{T} U_{1} R_{4}=U_{1}$ and $R_{4}^{T} U_{2} R_{4}=U_{4}, R_{7}^{T} U_{1} R_{7}=U_{2}$ and $R_{7}^{T} U_{2} R_{7}=U_{3}$, and $R_{3}^{T} U_{1} R_{3}=U_{3}$ and $R_{3}^{T} U_{2} R_{3}=U_{4}$.

\section{The STABiLity AND UNIQUENESS OF THE MiCROSTRUCTURE}

In the previous section, we proved the estimate

$$
\tau_{k}(y) \leq C\left[\mathcal{E}(y)^{1 / 2}+\mathcal{E}(y)\right] \quad \text { for } k \in\{1, \ldots, 4\} \backslash\{i, j\} \text { and all } y \in \mathcal{A},
$$

for all of the tetragonal to monoclinic transformations except when the lattice parameters satisfy the identities given in Theorem 3.1. We recall that

$$
\mathcal{A}=\left\{y \in W^{\phi}: y(x)=y_{0}(x) \text { for } x \in \partial \Omega\right\}
$$

where

$$
y_{0}(x)=\left[\lambda Q U_{i}+(1-\lambda) U_{j}\right] x \quad \text { for all } x \in \Omega .
$$

The results in this section for the tetragonal to monoclinic transformations can be deduced from the inequality (4.1) by the identical arguments used to deduce the results from (4.1) for the cubic to orthorhombic case [6] by making the obvious modifications in the argument to change $N=6$ to $N=4$. For this reason, we state the results given in this section without proof.

We also recall that the energy density $\phi$ is minimized on the union $\mathcal{U}$ of the four energy wells

$$
\mathcal{U}_{i}=\mathrm{SO}(3) U_{i}=\left\{R U_{i}: R \in \mathrm{SO}(3)\right\} \quad \text { for } i=1, \ldots, 4,
$$

and that $\phi$ is continuous and satisfies the growth condition

$$
\phi(F) \geq \kappa\|F-\pi(F)\|^{2} \quad \text { for all } F \in \mathbb{R}^{3 \times 3} .
$$


We shall also assume that the lattice parameters do not satisfy the identities given in Lemma 3.1 so that the inequality (4.1) holds.

Our first theorem in this section gives estimates for the derivative of the limiting macroscopic deformation $y$ in any direction tangential to the parallel layers of the laminate, for the $\mathrm{L}^{2}$ approximation of the limiting macroscopic deformation, and for the weak convergence of the limiting macroscopic deformation.

Theorem 4.1. (1) For any $w \in \mathbb{R}^{3}$ such that $w \cdot n=0$ and $|w|=1$, we have

$$
\int_{\Omega}\left|\left[\nabla y(x)-\nabla y_{0}(x)\right] w\right|^{2} \mathrm{~d} x \leq C\left[\mathcal{E}(y)^{1 / 2}+\mathcal{E}(y)\right] \quad \text { for all } y \in \mathcal{A} .
$$

(2) We have

$$
\int_{\Omega}\left|y(x)-y_{0}(x)\right|^{2} \mathrm{~d} x \leq C\left[\mathcal{E}(y)^{1 / 2}+\mathcal{E}(y)\right] \quad \text { for all } y \in \mathcal{A} .
$$

(3) For any Lipschitz domain $\omega \subset \Omega$, there exists a constant $C=C(\omega)>0$ such that

$$
\left\|\int_{\omega}\left[\nabla y(x)-\nabla y_{0}(x)\right] \mathrm{d} x\right\| \leq C\left[\mathcal{E}(y)^{1 / 8}+\mathcal{E}(y)^{1 / 2}\right] \quad \text { for all } y \in \mathcal{A} .
$$

The following corollary shows that the deformation gradients of energy-minimizing sequences of deformations must oscillate with a length scale that converges to zero.

Corollary 4.1. There does not exist any $y \in \mathcal{A}$ such that

$$
\mathcal{E}(y)=\min _{z \in \mathcal{A}} \mathcal{E}(z)
$$

For fixed $i, j \in\{1, \ldots, 4\}$ with $i \neq j$, we can define a projection $\pi_{i j}: \mathbb{R}^{3 \times 3} \rightarrow \mathcal{U}_{i} \cup \mathcal{U}_{j}$ by

$$
\left\|F-\pi_{i j}(F)\right\|=\min _{G \in \mathcal{U}_{i} \cup \mathcal{U}_{j}}\|F-G\| \quad \text { for all } F \in \mathbb{R}^{3 \times 3} .
$$

We also define the operators $\Theta: \mathbb{R}^{3 \times 3} \rightarrow \mathrm{SO}(3)$ and $\Pi: \mathbb{R}^{3 \times 3} \rightarrow\left\{Q U_{i}, U_{j}\right\}$ by the unique decomposition

$$
\pi_{i j}(F)=\Theta(F) \Pi(F) \quad \text { for all } F \in \mathbb{R}^{3 \times 3} .
$$

The following theorem proves that the deformation gradients of energy-minimizing sequences of deformations must oscillate between $Q U_{i}$ and $U_{j}$.

Theorem 4.2. We have

$$
\int_{\Omega}\|\nabla y(x)-\Pi(\nabla y(x))\|^{2} \mathrm{~d} x \leq C\left[\mathcal{E}(y)^{1 / 2}+\mathcal{E}(y)\right] \quad \text { for all } y \in \mathcal{A} .
$$

For any subset $\omega \subset \Omega, \rho>0$, and $y \in \mathcal{A}$, we define the sets

$$
\begin{aligned}
& \omega_{\rho}^{i}(y)=\left\{x \in \omega: \Pi(\nabla y(x))=Q U_{i} \text { and }\left\|\nabla y(x)-Q U_{i}\right\|<\rho\right\}, \\
& \omega_{\rho}^{j}(y)=\left\{x \in \omega: \Pi(\nabla y(x))=U_{j} \text { and }\left\|\nabla y(x)-U_{j}\right\|<\rho\right\} .
\end{aligned}
$$

The next theorem demonstrates that the deformation gradients of energy-minimizing sequences of deformations must oscillate with local volume fraction $\lambda$ at $Q U_{i}$ and local volume fraction $1-\lambda$ at $U_{j}$. It also demonstrates that the Young measure for this problem is unique $[3,28]$ and is given by

$$
\nu=\lambda \delta_{Q U_{i}}+(1-\lambda) \delta_{U_{j}} .
$$


Theorem 4.3. For any Lipschitz domain $\omega \subset \Omega$ and any $\rho>0$, there exists a constant $C=C(\omega, \rho)>0$ such that for all $y \in \mathcal{A}$

$$
\left|\frac{\operatorname{meas} \omega_{\rho}^{i}(y)}{\operatorname{meas} \omega}-\lambda\right|+\left|\frac{\operatorname{meas} \omega_{\rho}^{j}(y)}{\operatorname{meas} \omega}-(1-\lambda)\right| \leq C\left[\mathcal{E}(y)^{1 / 8}+\mathcal{E}(y)^{1 / 2}\right]
$$

We now denote by $\mathcal{V}$ the Sobolev space of all measurable functions $f: \Omega \times \mathbb{R}^{3 \times 3} \rightarrow \mathbb{R}$ such that

$$
\|f\|_{\mathcal{V}}^{2}=\int_{\Omega}\left\{\left[\operatorname{essipp}_{F \in \mathbb{R}^{3 \times 3}}\left\|\nabla_{F} f(x, F)\right\|\right]^{2}+\left|\nabla z_{f}(x) n\right|^{2}+z_{f}(x)^{2}\right\} \mathrm{d} x<\infty
$$

where $z_{f}: \Omega \rightarrow \mathbb{R}$ is defined by

$$
z_{f}(x)=f\left(x, Q U_{i}\right)-f\left(x, U_{j}\right) \quad \text { for all } x \in \Omega
$$

The final theorem in this section gives an estimate for the weak convergence of nonlinear functions of the deformation gradient.

Theorem 4.4. We have

$$
\begin{aligned}
& \mid \int_{\Omega}\{f(x,\left.\nabla y(x))-\left[\lambda f\left(x, Q U_{i}\right)+(1-\lambda) f\left(x, U_{j}\right)\right]\right\} \mathrm{d} x \mid \\
& \leq C\|f\|_{\mathcal{V}}\left[\mathcal{E}(y)^{1 / 4}+\mathcal{E}(y)^{1 / 2}\right] \quad \text { for all } f \in \mathcal{V} \text { and all } y \in \mathcal{A}
\end{aligned}
$$

\section{The Finite ELEMENT APPROXIMATION OF MICROSTRUCTURE}

The simplest finite element approximation of the variational problem

$$
\inf _{v \in \mathcal{A}} \mathcal{E}(v)
$$

is given by

$$
\inf _{v_{h} \in \mathcal{A}_{h}} \mathcal{E}\left(v_{h}\right)
$$

where $\mathcal{A}_{h}$ is a finite-dimensional subspace of $\mathcal{A}$ defined for $h \in\left(0, h_{0}\right]$ for some $h_{0}>0$. The following approximation theorem for the energy can be proven for the most widely used $P_{k}$ or $Q_{k}$ type conforming finite elements on quasi-regular meshes, in particular for the $P_{1}$ linear elements defined on tetrahedra and the $Q_{1}$ trilinear elements defined on rectangular parallelepipeds [6,12,23-25, 27,28].

Theorem 5.1. For each $h \in\left(0, h_{0}\right]$, there exists $y_{h} \in \mathcal{A}_{h}$ such that

$$
\mathcal{E}\left(y_{h}\right)=\min _{z_{h} \in \mathcal{A}_{h}} \mathcal{E}\left(z_{h}\right) \leq C h^{1 / 2}
$$

For the remainder of this section, we again recall that the energy density $\phi$ is minimized on the union $\mathcal{U}$ of the four energy wells

$$
\mathcal{U}_{i}=\operatorname{SO}(3) U_{i}=\left\{R U_{i}: R \in \operatorname{SO}(3)\right\} \quad \text { for } i=1, \ldots, 4,
$$

and that $\phi$ is continuous and satisfies the growth condition

$$
\phi(F) \geq \kappa\|F-\pi(F)\|^{2} \quad \text { for all } F \in \mathbb{R}^{3 \times 3} .
$$


We shall also assume that the lattice parameters do not satisfy the identities given in Lemma 3.1 so that the results of the previous section hold. In this case, the following corollaries for the finite element approximation follow directly from the above estimate for the approximation of the energy (5.1). We assume below that $y_{h} \in \mathcal{A}_{h}$ is a finite element approximation satisfying the quasi-optimality condition

$$
\mathcal{E}\left(y_{h}\right) \leq \sigma \inf _{z_{h} \in \mathcal{A}_{h}} \mathcal{E}\left(z_{h}\right)
$$

for some constant $\sigma \geq 1$ independent of $h$.

\section{Corollary 5.1.}

(1) There exists a positive constant $C$ such that for any $y_{h} \in \mathcal{A}_{h}$ satisfying (5.2) we have

$$
\int_{\Omega}\left|y_{h}(x)-y_{0}(x)\right|^{2} \mathrm{~d} x \leq C h^{1 / 4}
$$

and

$$
\int_{\Omega}\left\|\nabla y_{h}(x)-\Pi\left(\nabla y_{h}(x)\right)\right\|^{2} \mathrm{~d} x \leq C h^{1 / 4} .
$$

(2) For any $w \in \mathbb{R}^{3}$ such that $w \cdot n=0$ and $|w|=1$, we have

$$
\int_{\Omega}\left|\left[\nabla y_{h}(x)-\nabla y_{0}(x)\right] w\right|^{2} \mathrm{~d} x \leq C h^{1 / 4}
$$

for any $y_{h} \in \mathcal{A}_{h}$ satisfying (5.2).

(3) If $\omega \subset \Omega$ is a Lipschitz domain, then there exists a constant $C=C(\omega)>0$ such that for any $y_{h} \in \mathcal{A}_{h}$ satisfying (5.2) we have

$$
\left\|\int_{\omega}\left[\nabla y_{h}(x)-\nabla y_{0}(x)\right] \mathrm{d} x\right\| \leq C h^{1 / 16} .
$$

Corollary 5.2. (1) If $\omega \subset \Omega$ is a Lipschitz domain and $\rho>0$, then there exists a constant $C=C(\omega, \rho)>0$ such that for any $y_{h} \in \mathcal{A}_{h}$ satisfying (5.2)

$$
\left|\frac{\operatorname{meas} \omega_{\rho}^{i}\left(y_{h}\right)}{\operatorname{meas} \omega}-\lambda\right|+\left|\frac{\operatorname{meas} \omega_{\rho}^{j}\left(y_{h}\right)}{\operatorname{meas} \omega}-(1-\lambda)\right| \leq C h^{1 / 16} .
$$

(2) We have

$$
\left|\int_{\Omega}\left\{f\left(x, \nabla y_{h}(x)\right)-\left[\lambda f\left(x, Q U_{i}\right)+(1-\lambda) f\left(x, U_{j}\right)\right]\right\} \mathrm{d} x\right| \leq C\|f\|_{\mathcal{V}} h^{1 / 8}
$$

for any $f \in \mathcal{V}$ and any $y_{h} \in \mathcal{A}_{h}$ satisfying (5.2).

\section{REFERENCES}

[1] R. Adams. Sobolev Spaces. Academic Press, New York (1975).

[2] J. Ball and R. James, Fine phase mixtures as minimizers of energy. Arch. Rat. Mech. Anal. 100 (1987) 13-52.

[3] J. Ball and R. James, Proposed experimental tests of a theory of fine microstructure and the two-well problem. Phil. Trans. R. Soc. Lond. A 338 (1992) 389-450. 
[4] K. Bhattacharya, Self accomodation in martensite. Arch. Rat. Mech. Anal. 120 (1992) 201-244.

[5] K. Bhattacharya and G. Dolzmann, Relaxation of some multiwell problems, in Proc. R. Soc. Edinburgh: Section A, to appear.

[6] K. Bhattacharya, B. Li and M. Luskin, The simply laminated microstructure in martensitic crystals that undergo a cubic to orthorhombic phase transformation. Arch. Rat. Mech. Anal. 149 (2000) 123-154.

[7] B. Brighi and M. Chipot, Approximation of infima in the calculus of variations. J. Comput. Appl. Math. 98 (1998) $273-287$.

[8] C. Carstensen and P. Plecháč, Numerical solution of the scalar double-well problem allowing microstructure. Math. Comp., 66 (1997) 997-1026.

[9] C. Carstensen and P. Plecháč, Adaptive algorithms for scalar non-convex variational problems. Appl. Numer. Math. 26 (1998) 203-216.

[10] M. Chipot, Numerical analysis of oscillations in nonconvex problems. Numer. Math. 59 (1991) $747-767$.

[11] M. Chipot and C. Collins, Numerical approximations in variational problems with potential wells. SIAM J. Numer. Anal. 29 (1992) 1002-1019.

[12] M. Chipot, C. Collins, and D. Kinderlehrer, Numerical analysis of oscillations in multiple well problems. Numer. Math. 70 (1995) 259-282.

[13] M. Chipot and D. Kinderlehrer, Equilibrium configurations of crystals. Arch. Rat. Mech. Anal. 103 (1988) $237-277$.

[14] M. Chipot and S. Müller, Sharp energy estimates for finite element approximations of nonconvex problems. (preprint, 1997).

[15] C. Collins, D. Kinderlehrer, and M. Luskin, Numerical approximation of the solution of a variational problem with a double well potential. SIAM J. Numer. Anal. 28 (1991) 321-332.

[16] C. Collins and M. Luskin, Optimal order estimates for the finite element approximation of the solution of a nonconvex variational problem. Math. Comp. 57 (1991) 621-637.

[17] B. Dacorogna, Direct methods in the calculus of variations. Springer-Verlag, Berlin, (1989).

[18] G. Dolzmann, Numerical computation of rank-one convex envelopes. SIAM J. Numer. Anal. 36 (1999) $1621-1635$.

[19] D. French, On the convergence of finite element approximations of a relaxed variational problem. SIAM J. Numer. Anal. 28 (1991) 419-436.

[20] L. Jian and R. James, Prediction of microstructure in monoclinic $\mathrm{LaNbO}_{4}$ by energy minimization. Acta Mater. 45 (1997) 4271-4281.

[21] D. Kinderlehrer and P. Pedregal, Characterizations of gradient Young measures. Arch. Rat. Mech. Anal. 115 (1991) $329-365$.

[22] M. Kružík, Numerical approach to double well problems. SIAM J. Numer. Anal. 35 (1998) $1833-1849$.

[23] B. Li and M. Luskin, Finite element analysis of microstructure for the cubic to tetragonal transformation. SIAM J. Numer. Anal. 35 (1998) 376-392.

[24] B. Li and M. Luskin, Nonconforming finite element approximation of crystalline microstructure. Math. Comp. 67(223) (1998) 917-946.

[25] B. Li and M. Luskin, Approximation of a martensitic laminate with varying volume fractions. Math. Model. Numer. Anal. 33 (1999) 67-87.

[26] Z. Li, Simultaneous numerical approximation of microstructures and relaxed minimizers. Numer. Math. 78 (1997) 21-38.

[27] M. Luskin, Approximation of a laminated microstructure for a rotationally invariant, double well energy density. Numer. Math. 75 (1996) 205-221.

[28] M. Luskin, On the computation of crystalline microstructure. Acta Numer. (1996) 191-257.

[29] M. Luskin and L. Ma, Analysis of the finite element approximation of microstructure in micromagnetics. SIAM J. Numer. Anal. 29 320-331.

[30] R. Nicolaides and N. Walkington, Strong convergence of numerical solutions to degenerate variational problems. Math. Comp. 64 (1995) 117-127.

[31] P. Pedregal, Numerical approximation of parametrized measures. Num. Funct. Anal. Opt. 16 (1995) $1049-1066$.

[32] P. Pedregal, On the numerical analysis of non-convex variational problems. Numer. Math. 74 (1996) 325-336.

[33] T. Roubíček, Numerical approximation of relaxed variational problems. J. Convex Anal. 3 (1996) 329-347.

[34] N. Simha, Crystallography of the tetragonal $\rightarrow$ monoclinic transformation in zirconia. J. Phys. IV Colloq. France 5 (1995) C81121-C81126.

[35] N. Simha, Twin and habit plane microstructures due to the tetragonal to monoclinic transformation of zirconia. J. Mech. Phys. Solids 45 (1997) 261-292.

[36] V. Šverák, Lower-semicontinuity of variational integrals and compensated compactness, in Proceedings ICM 94, Zürich (1995). Birkhäuser.

[37] L. Tartar, Compensated compactness and applications to partial differential equations, in: Nonlinear analysis and mechanics, R. Knops, Ed., Pitman Research Notes in Mathematics, London 39 (1978) 136-212.

[38] G. Zanzotto, Twinning in minerals and metals: remarks on the comparison of a thermoelasticity theory with some available experimental results. Atti Acc. Lincei Rend. Fis. 82 (1988) 725-756. 